\title{
Aportaciones de la Educación en Derechos Humanos (EDH) y la Investigación Acción Participativa (IAP) en contextos de trabajo comunitario
}

\author{
Carla Cubillos-Vega \\ Trabajadora Social. Doctora en Trabajo Social \\ Universidad Complutense de Madrid. Madrid, España \\ https://orcid.org/0000-0002-3306-8787 • carlacubillos@gmail.com
}

\section{Resumen}

En este trabajo se explora la conexión entre la dimensión comunitaria, política y ética del Trabajo Social, la educación en derechos humanos y las prácticas participativas, en concreto la Investigación acción participativa, coincidiendo con un objetivo común: facilitar la emancipación de las personas y grupos. Para ello se indagan las posibilidades de la Educación en Derechos Humanos crítica y la Investigación Acción Participativa como estrategias a utilizar en el marco de la práctica del Trabajo Social con comunidades en el ámbito de la promoción y defensa de los derechos humanos. De este modo, se exploran las posibilidades concretas de promoción, defensa y exigibilidad de los derechos humanos en y por las comunidades. Dichas estrategias se presentan, así, como herramientas para aterrizar los derechos humanos al plano de lo cotidiano.

Palabras clave: Trabajo Social; Derechos Humanos; Educación; InvestigaciónAcción Participativa; Trabajo comunitario.

Recibido: 04/07/2019 | Aprobado: 15/10/2019 


\title{
Contributions of Human Rights Education (HRE) and Participatory Action Research (PAR) in Contexts of Community Work
}

\begin{abstract}
This work explores the community, political, and ethical dimensions of Social Work and their connection with human rights education and participatory practices, particularly participatory action research, and their confluence in a common goal: promoting the emancipation of people and groups. The study explores critical human rights education and participatory action research as strategies to be used in the practice of Social Work with communities in the defense and promotion of human rights. Thus, realistic possibilities to promote, defend and enforce human rights in and by the communities are explored. These strategies are presented together with tools to integrate human rights in quotidian life.

Keywords: Social work; Human rights; Education; Participatory Action Research; Community work.

Sumario: 1. Introducción, 2. La EDH y el modelo de la transformación, 3. El método de la Investigación acción participativa (IAP), 4. El punto de convergencia, 5. Conclusiones, 6. Referencias bibliográficas.
\end{abstract}




\section{Introducción}

El Trabajo Social no se puede desvincular de la política en tanto se ejerce en un contexto de políticas, contribuye a canalizar disposiciones o medidas de políticas sociales (Zamanillo y Martín, 2011) -en muchos contextos nacionales el lobby realizado por trabajadores/as sociales contribuye a producir sendos cambios en las políticas públicas (Beydili \& Yildirim, 2013; Nadkarni \& Sinha, 2016). Su nacimiento, como profesión, se cimentó en la implicación política de varias de las madres de la profesión (Cubillos-Vega, 2019), todo ello sin perjuicio de que en la actualidad y en muchos contextos nuestras prácticas profesionales se han visto burocratizadas y estandarizadas al desarrollarse en el seno de organizaciones complejas, y en otros casos aún peores, se orientan al mero asistencialismo, allí donde no hay políticas de bienestar o las que hay, se enmarcan en la doctrina neoliberal.

El Trabajo Social nace, como profesión, en un contexto de reforma social vinculado a la devastación social que trajo consigo el proceso de industrialización, primero en el Reino Unido (RU), expandiéndose luego al resto de Europa y los Estados Unidos de América (EE.UU.). Martínez y Agüero (2008) afirman que "no sólo su origen se vincula a un proyecto político conservador de carácter imperialista, sino también su desarrollo y expansión al resto de Europa y Estados Unidos" (p. 20) pues la impronta política en el desarrollo del Trabajo Social se debió a que este fue creado e instrumentalizado política e ideológicamente por parte de los grupos políticos y económicos hegemónicos, usándolo para el control social de la fuerza de trabajo y, en definitiva, para la acumulación del capital.

No obstante, dicha interpretación queda estrecha si consideramos que, pese a que en un inicio el Trabajo Social nace en el RU como una práctica orientada a la moralización y el control social, fue en EEUU donde las madres fundadoras del Trabajo Social, vinculadas a la Hull House y a la Escuela sociológica de Chicago, impulsaron importantes reformas sociales en respuesta a la crisis arriba mencionada; no podemos ignorar que la Hull House, dirigida por Jane Addams, fue un verdadero centro comunitario con un amplio catálogo de actividades orientadas a promover la emancipación de las personas (Miranda, 2003; 2014).

En todo caso, sea que nos decantemos, bien por la identidad del Trabajo Social como un instrumento político al servicio de los poderes económicos, bien con la de agentes políticos al servicio de la transformación social contra las injusticias, o bien adoptando una perspectiva matizada, no cabe duda de que en ambas visiones se evidencia, como señala Duarte (2012), su rol profesional como actores políticos que realizan praxis política y que actúan en un mundo político. Así, de acuerdo con Zamanillo y Martín (2011), el Trabajo Social "no puede estar ajeno a la política. La política le concierne, tanto en la comprensión de la dimensión estructural de las desigualdades sociales, como en las decisiones que ha de tomar [en su ejercicio]" (p. 103) a lo cual cabría añadir que también le concierne en virtud de las acciones que puede emprender como colectivo organizado supervisando y/o exigiendo políticas sociales adecuadas a esa realidad que conoce de primera mano. 
Cubillos-Vega

Es en dicha faceta política -y ética-, que el Trabajo Social conecta con la educación en derechos humanos (EDH), como estrategia pedagógica crítica, y con prácticas participativas como es el caso de la Investigación acción participativa (IAP), compartiendo un mismo objetivo: facilitar la emancipación de las personas para que estas participen activamente en la transformación de la realidad social y la consecución de su bienestar. La mayoría de las veces, dicho bienestar se va a traducir en el ejercicio y disfrute efectivo de sus derechos, cuya mayor barrera se encuentra en el desconocimiento de los mismos.

En este trabajo se van a indagar las posibilidades que ofrecen al Trabajo Social Comunitario, la EDH crítica y la IAP como herramientas para aterrizar los derechos humanos al plano de lo cotidiano y explorar sus posibilidades de realización efectiva en las comunidades de acuerdo a sus propios códigos culturales y sus recursos.

\section{La EDH y el modelo de la transformación}

Para que la protección de los derechos humanos pueda ser efectiva -y aunque sea más una utopía, alcanzar un plano universal-, esta solo puede realizarse mediante la información y la demanda continua de dicha protección a los Estados por parte de las personas y grupos; solo en la medida en que estas/os conozcan sus derechos y los medios para exigir su respeto, será posible realizarlos (UNESCO, 2000). De ahí que la EDH constituye en sí misma un derecho. Pese a que en la actualidad es muy poco utilizada por quienes se ocupan de orientar, fomentar o dinamizar estas prácticas (Mihr \& Schmitz, 2007), la EDH es una poderosa estrategia al servicio del activismo y de los procesos de transformación social orientados a la prevención de violaciones de derechos humanos, así como a la promoción de nuevos derechos y al reclamo de aquellos aun no garantizados o efectivamente protegidos.

La EDH es un proceso educativo en cuya definición toma como base un enfoque histórico y amplio de los derechos humanos, vinculado con nociones de justicia, paz, pluralidad y democracia; el objetivo es educar en la práctica de los derechos humanos y en su defensa a través del desarrollo de una conciencia crítica que promueva un cambio estructural, sobre cuestiones como la defensa de la dignidad, la libertad, la justicia y la equidad, lejos de una lógica formal de educación bancaria y de modelos educativos verticales o autoritarios y traspasando las instancias educativas formales, dirigiéndose a la educación de toda la sociedad (Cubillos-Vega, 2018; Rojas, 2015).

La EDH en contextos de educación no formal dirigida a personas adultas, está estrechamente relacionada con la educación popular. Son varias las evidencias que en una dimensión histórica e internacional podemos encontrar sobre la utilización de la EDH como una herramienta liberalizadora en contextos opresivos, pudiendo situar su origen precisamente a partir de los movimientos sociales y la educación popular (Magendzo, 2015). La educación popular es una tradición político-pedagógica crítica (Muñoz, 2013) que se origina en la década de los 60 en el marco de la conmoción política que afectaba a diversos países en Sudamérica y el Caribe donde los derechos humanos se estaban violando gravemente (Zúñiga, 2018). En el marco de las dictaduras de los años 70 en Latinoamérica, a partir de la década de los 80 , fue utilizada como una herramienta para el cambio social no violento y la redemocratización de la sociedad, especialmente en Argentina, Brasil, Chile y

Prospectiva. Revista de Trabajo Social e intervención social • No. $29 \bullet$ ene.jun. 2020 • pp. 173-192 e-ISSN: 2389-993X • Doi: 10.25100/prts.v0i29.8224

Universidad del Valle - Cali, Colombia 
Perú, donde las/los educadores activistas usaron la educación popular en derechos humanos para acercarse a las personas con programas educativos que condenaban las violaciones de derechos y aclamaban la restauración de la democracia, de modo que con el reconocimiento de las organizaciones, se convirtió en un componente esencial de la resistencia popular (Cubillos-Vega y Llanán, 2018; Flowers, 2015; Magendzo y Bermúdez, 2018). Pero el foco no solo se ha situado en la vindicación de derechos políticos y civiles, sino también de derechos sociales y, en suma, de justicia social.

Cuando se desarrolla en contextos no formales, la EDH, es educación popular en derechos humanos, tal como subraya Claude (2003), para quien la educación popular se dirige a las comunidades de base con el objeto de desarrollar su conocimiento sobre sus derechos y su capacidad legal para defenderlos y exigirlos; esto último, nos dice, constituiría la herramienta con la cual los sujetos y comunidades pueden aumentar el control de sus vidas y las decisiones que les afectan, en un continuo proceso de exploración y reflexión crítica.

En Latinoamérica, la educación popular en derechos humanos resurge en la década de los 90, especialmente en Argentina, como un medio para manifestarse en contra de las reformas neoliberales impulsadas por los gobiernos latinoamericanos, reivindicando el sector público y generando nuevas alternativas a los procesos de marginalización social y de múltiples pobrezas o necesidades (de protección, de pensamiento reflexivo y de participación política y social) que las políticas neoliberales -y las reformas estructurales realizadas en base a las mismas- estaban generando (Ampudia, 2012; Elisalde y Ampudia, 2008). Las iniciativas desarrolladas -como el bachillerato popular- surgen como nuevos espacios contra hegemónicos promovidos por movimientos y organizaciones sociales con el objeto de fortalecer el trabajo territorial en los barrios mediante la Educación Popular (Ampudia, 2012). Igualmente, en el siglo XXI, la educación popular ha integrado a su propuesta las ideas del movimiento feminista, por la paz y las autonomías de los pueblos originarios (Zúñiga, 2018). De acuerdo con Ventura (2018), en los "procesos de educación popular en derechos humanos" (p. 28) se busca fortalecer el vínculo con los movimientos sociales y con los colectivos que han sido discriminados estructuralmente a partir de la reflexión colectiva crítica en cuyo ejercicio lo cotidiano es parte de la lucha política.

Tanto el enfoque de EDH crítico como la educación popular (una de sus manifestaciones cuando se dirige a personas adultas en contextos no formales), beben de las teorías de la pedagogía crítica de P. Freire y su obra Pedagogía del Oprimido (Flowers, 2015; Magendzo y Bermúdez, 2018). A su vez, el pensamiento de Freire y los desarrollos posteriores, estuvieron ampliamente influenciados por la Educación Popular europea, promovida por Condorcet (Mejía, 2011), por la Teoría Crítica de la Escuela de Frankfurt (Brito, 2008), por la noción de "emancipación" acuñada por Adorno (1998) en la década de los 60, así como por las ideas de la nueva sociología de la educación, cuya cuna se situó en Inglaterra y EE.UU en la década de los 70 (Freire, 1990), así como por los trabajos de J. Dewey $^{1}$ quien, a inicios del S.XX, criticaba férreamente la educación orientada al

${ }^{1}$ Este filósofo y pedagogo estadounidense, mientras estuvo adscrito a la Escuela Sociológica de Chicago, solía frecuentar la Hull House, entonces dirigida por Jane Addams que, además de centro

Prospectiva. Revista de Trabajo Social e intervención social • No. 29 • ene.-jun. 2020 • pp. 173-192 e-ISSN: 2389-993X • Doi: 10.25100/prts.v0i29.8224

Universidad del Valle - Cali, Colombia 
Cubillos-Vega

almacenamiento del conocimiento (Dewey, 2002), una crítica que luego Freire desarrollaría con su noción original de educación bancaria (Freire, 2012). De este modo, en Latinoamérica se ha venido construyendo un pensamiento propio y original que, aunque nutrido de fuentes euroamericanas y en constante diálogo con las mismas, tiene sus particularidades propias de los contextos, culturas y saberes del Sur (Mejía, 2011).

En el caso de España, la EDH surge y se desarrolla en el marco de la instauración de la democracia de la mano, igualmente, de organizaciones no gubernamentales, entre las que cabe destacar la Sección española de Amnistía Internacional y la Asociación Pro Derechos Humanos de España (Tuvilla, 2008). Así mismo, inspirados en la EDH desarrollada en Latinoamérica, Flowers (2015) nos relata cómo las/los jóvenes activistas en Filipinas, en los años 80, fueron a los barrios marginales y las zonas rurales para enseñar los derechos humanos a pescadores, agricultores y personas pobres de las zonas urbanas, contribuyendo directamente a la "revolución del poder del pueblo", una campaña sostenida de resistencia popular y no violencia que culminó con el derrocamiento del régimen autoritario de F. Marcos.

La EDH no debe plantearse como una mera información teórica, sino que debe contribuir a concientizar a las personas sobre cómo aplicar, promover y defender los derechos humanos en sus vidas en tanto instrumentos para alcanzar una vida digna. Tal como señala la Asamblea General de Naciones Unidas (2017) en el informe de la Mesa redonda sobre la aplicación de la Declaración de las Naciones Unidas sobre Educación y Formación en materia de Derechos Humanos, la EDH debería favorecer el pensamiento crítico y sensibilizar a las personas sobre la adopción de soluciones inclusivas, propiciando su participación. En dicha sesión se proyectó parte del documental A Path to Dignity: The Power of Human Rights Education ${ }^{2}$ donde se relatan tres historias de tres mujeres víctimas de distintos tipos de discriminación y violencias, participantes en programas de EDH en diferentes lugares del mundo, quienes relatan cómo en el proceso educativo se fueron concientizando de su problema y empoderando, comenzando a verse ya no como víctimas, sino como activistas por la defensa de los derechos humanos. Las conclusiones que destacó la moderadora de la mesa fueron las siguientes:

a) la educación en materia de derechos humanos comenzaba con la vida cotidiana de los educandos, haciendo que participasen en una reflexión crítica sobre los problemas de derechos humanos a que se enfrentaban; b) la educación en materia de derechos humanos era un proceso de empoderamiento que dotaba a los educandos de instrumentos, conocimientos y aptitudes para resolver sus problemas de derechos humanos; y c) la educación en materia de derechos humanos daba lugar a la adopción de medidas en consonancia con los principios básicos de los derechos humanos a los efectos de promover y proteger tales derechos. (Asamblea General de Naciones Unidas, 2017, p. 4).

Vemos que en la actualidad no es raro que se aluda al carácter local y preventivo de la EDH centrada en el activismo y la labor de los movimientos sociales; Mihr y Schmitz

comunitario, era un espacio de reunión para el debate entre anarquistas, marxistas, socialistas, unionistas, sociólogos y profesores de la Universidad de Chicago (Miranda, 2003).

$2 \mathrm{Al}$ cual se puede acceder siguiendo este enlace: http://www.ohchr.org/EN/NewsEvents/Pages/ThePowerofHumanRightsEducation.aspx.

Prospectiva. Revista de Trabajo Social e intervención social • No. $29 \bullet$ ene.jun. 2020 • pp. 173-192 e-ISSN: 2389-993X • Doi: 10.25100/prts.v0i29.8224 Universidad del Valle - Cali, Colombia 
(2007) coincidiendo con los enfoques latinoamericanos, defienden que dicho activismo debe enfatizar el significado original de los derechos humanos como una estrategia de emancipación individual y, en extensión, de las comunidades; para ello, añaden, las Organizaciones de defensa de los derechos humanos deben cooperar y generar estrategias más sostenibles con las comunidades locales, contribuyendo a fortalecer a los/las participantes en el proceso de definición de los desafíos sociales y políticos a los que se enfrentan. Como estos autores señalan, el conocimiento de los derechos humanos por sí solo no previene las violaciones de derechos, ni empodera por si solo a las víctimas, ni alienta al resto de personas a intervenir; es preciso que las personas definan por sí mismas cuáles son sus necesidades y cómo los valores de los derechos humanos pueden contribuir a su bienestar; la EDH debe reflejar un proceso interactivo, por lo tanto la EDH es un proceso intrínsecamente abierto, centrado principalmente en acabar con las violaciones de derechos y prevenirlas en el futuro (Mihr y Schmitz, 2007).

Con todo este encuadre, podemos apreciar que, en lo teórico, el marco de cambio social al que aspira la EDH se caracteriza principalmente por el fomento del pensamiento crítico, el diálogo, la emancipación personal y colectiva y, a largo plazo, la justicia social (Hawkins \& Knox, 2014; Magendzo, 2003; Magendzo y Bermúdez, 2018; Tibbitts, 2002; Ventura, 2018); todo ello a través de la exigibilidad de derechos que han sido construidos históricamente gracias a las luchas de los pueblos y de diversos movimientos sociales

Respecto a la práctica de la EDH, además de las numerosas experiencias sistematizadas de EDH latinoamericanas, algunas autoras anglosajonas han ido identificando modelos o tipologías de $\mathrm{EDH}$ que nos son útiles para definir objetivos adecuados a cada tipo de audiencia. Por ejemplo, F. Tibbitts (2002; 2017) en su famosa tipología de los tres modelos de EDH clasificados según la audiencia a la cual esta va dirigida y los objetivos a alcanzar, identifica: (1) el modelo de los valores, que se dirige al público general y muy en especial al alumnado de educación formal, el cual está orientado a la socialización; (2) el modelo de la responsabilidad, dirigido a profesionales con incidencia en el ámbito de los derechos humanos y enfocado al desarrollo profesional en dicha materia; y (3) el modelo de la transformación, cuyo objetivo es el paso desde la información/sensibilización hacia la acción.

Este modelo prepara a las personas para comprender que su situación es producto de una vulneración sistemática de sus derechos y tomar, en consecuencia, cauces de acción. Dentro de este modelo podríamos situar, por ejemplo, los casos testimoniados en el documental comentado arriba. El modelo de transformación implica, como su nombre lo indica, un plano mayor de acción, en tanto que se prepara a la audiencia para comprometerse activamente por los derechos humanos; los programas se planifican con el objeto de empoderar a las personas, grupos e incluso comunidades, facultándoles progresivamente para reconocer las violaciones de sus derechos y comprometerse en prevenirlas. Se asume que las personas que integran la audiencia han sufrido dicha vulneración y por lo tanto tienen una mayor predisposición a convertirse en promotoras de su defensa. Así, mientras en los dos primeros modelos la participación es un componente deseable, en el tercero es una condición sine qua non para alcanzar los objetivos.

Prospectiva. Revista de Trabajo Social e intervención social • No. 29 • ene.-jun. $2020 \bullet$ pp. 173-192 e-ISSN: 2389-993X • Doi: 10.25100/prts.v0i29.8224

Universidad del Valle - Cali, Colombia 
Cubillos-Vega

En la misma línea, Bajaj (2011) identifica tres tipos de EDH según el contenido ideológico de las acciones, las cuales clasifica en: (a) EDH para la ciudadanía global (que coincidiría con los modelos de los valores y de la responsabilidad de Tibbits); (b) EDH para la coexistencia (orientado a entornos de conflictos/luchas étnicas o civiles); y (c) EDH para la acción transformadora, orientado a generar un proceso de cambio significativo en las personas sobre la base de la concienciación y de la interpretación de los derechos humanos como una herramienta política para alcanzar la justicia social (que coincidiría con el modelo para la transformación de Tibbits).

\section{El método de la Investigación acción participativa (IAP)}

En su desarrollo, entre los años 1960-70 la educación popular fue confluyendo -y se fue configurando- con aportes de diversas propuestas sociales o campos de estudio dentro del paradigma emancipatorio, destacando entre ellos el de la Investigación Acción participativa (Muñoz, 2013; Ortiz y Borjas, 2008). La investigación acción participativa (IAP) es una filosofía y un procedimiento de trabajo e investigación que, tal como nos recuerda Fals-Borda (1991) uno de sus padres creadores, no se orienta únicamente a la investigación ni tampoco es meramente educación para personas adultas o acción sociopolítica, sino que integra esos tres aspectos a modo de fases no necesariamente consecutivas. De este modo entronca con la EDH y con el trabajo comunitario en sus facetas educativa y movilizadora. Sus objetivos serían

(1) el capacitar a los grupos y clases oprimidas a adquirir la suficiente creatividad y fuerza transformadora, las que se expresan a través de proyectos, acciones y luchas específicas; y (2) el producir y desarrollar un proceso de pensamiento socio-político con el cual las bases populares se puedan identificar. (Fals-Borda, 1991, p. 9).

Como método, procedimiento, o modalidad de trabajo e investigación, se realiza mediante la implicación activa de las personas afectadas por un determinado problema o situación colectiva, a través de un proceso participativo donde es la propia población, con el apoyo de agentes especializados, quien identifica sus necesidades o problemas y las posibilidades de actuación de cara a la transformación social. Se distinguen, entonces, dos tipos de agentes de cambio: externos a las poblaciones vulnerabilizadas (p.ej. perfiles académicos o técnicos) e internos o pertenecientes a las mismas, donde, en una dinámica dialéctica, los primeros aportan el saber académico y los segundos y el saber experiencial popular (Fals-Borda, 1991).

Esta propuesta surge en Latinoamérica como una reacción al uso "descabellado y amoral" de la ciencia como un componente de intervención social, cuando esta se desarrolla unilateralmente desde la perspectiva de la/el investigador asumiendo un conocimiento absoluto de la realidad, independiente y objetivo, controlando la situación objeto de estudio (Fals-Borda, 1993, p. 14). Como señala Fals-Borda la IAP no rompe con el cientificismo instrumental, sino que lo va a acercar a la racionalidad empírica (o cotidiana) a través de la participación constructiva, la que termina con el anclado binomio sujeto/objeto caracterizado por una relación vertical, convirtiéndolo en una relación horizontal sujeto/sujeto. 
El objeto observado pasa a constituirse en sujeto protagonista de la acción de investigar que al mismo tiempo es acción transformadora, manteniéndose igualmente el rol de la/el especialista o profesional investigador, quien domina las técnicas de análisis, pero replantándose el objetivo y las personas destinatarias de la investigación -en oposición al modelo de investigación realizada para un cliente que la demanda- (Alberich, 2008). No obstante, una relación simétrica u horizontal, nos advierte Rodríguez (1993), nunca será absoluta si consideramos que por el mero hecho de promover y coordinar un proyecto IAP dichas/os especialistas estarán implicados, no pudiéndose pretender una neutralidad absoluta; se trata, más bien, de un aprendizaje mutuo.

Entre las influencias clave en el surgimiento de este enfoque encontramos el trabajo de Marx y Engels, Gramsci y la Escuela de Frankfurt, junto con los planteamientos de algunos movimientos sociales como los ecológicos, el feminismo y principalmente los movimientos de liberación latinoamericanos, siendo el trabajo de Freire y su pedagogía popular, un referente indiscutible (Alberich, 2008; Healy, 2001; Rahman, 1991). Sin embargo, en palabras de Rahman (1991) el desarrollo de la IAP como ideología parece responder tanto a la crisis de la izquierda como a la de la derecha dado que, aunque los partidos de vanguardia han generado importantes cambios estructurales en varias situaciones, han emergido igualmente "nuevas formas de dominación de las masas" (p. 23). Una de ellas es el poder social sobre la producción/transmisión del conocimiento. Solo será posible la emancipación de las personas y comunidades en la medida en que la gente se movilice por sí misma, y no a través de la reproducción de una "conciencia avanzada" (en términos intelectuales), de conocimientos e ideales que no sean los suyos propios (Rahman, 1991, p. 24). Por ello la participación activa de las personas y grupos, y la adquisición de poder social para generar su propio conocimiento y concientización son fundamentales.

El proceso de la IAP se va a caracterizar, así, por su por carácter participativo y colectivo y su finalidad concientizadora y transformadora (De la Riva, 1993; López, 1993); su finalidad última, a través de sus técnicas, es la movilización de las personas "desde las bases hacia arriba y desde la periferia hasta los centros", para que los movimientos sociales que conformen luchen por la participación, por la justicia y por la igualdad (Fals-Borda, 1991, pp. 12-13).

La promoción de los procesos de cambios sociales se hará mediante el aprendizaje mutuo entre todos los actores implicados (Observatorio Internacional de Ciudadanía y Medio Ambiente Sostenible-CIMAS, 2009; De la Riva, 1993) para lo cual se organizará en una serie de fases concretas recurriéndose a diversas técnicas de tipo cualitativo y cuantitativo, así como otras dinámicas participativas. Para analizar la situación de partida se comienza por el conocimiento y la sensibilización de la población que será la protagonista del proceso transformativo (Basagoiti, Bru y Lorenzana, 2001), proceso que se desarrollará a través de un proyecto IAP. La investigación no es desarrollada por personas "expertas" ajenas sino por las propias poblaciones afectadas (en adelante actores), lo cual como señala López (1993) es en sí misma una acción, dirigida a rescatar y desarrollar el saber propio de los actores implicados. Para que los actores identifiquen su situación (su contexto y realidad global y sus causas y efectos), de cara a conocer la realidad para transformarla, es preciso recurrir a su memoria colectiva, a su experiencia y su historia común (De la Riva, 1993).

Prospectiva. Revista de Trabajo Social e intervención social • No. $29 \bullet$ ene.-jun. 2020 • pp. 173-192 e-ISSN: 2389-993X • Doi: 10.25100/prts.v0i29.8224 Universidad del Valle - Cali, Colombia 
Cubillos-Vega

Pese a que como el propio Fals-Borda (1991) ha advertido, las tareas sociopolíticas que implica el desarrollo de procesos IAP no pueden ser rigurosamente planificadas, ni generalizadas, ni mucho menos copiadas de manera acrítica y descontextualizada al suponer sistemas sociales abiertos y procesos coyunturales, a continuación se expone una propuesta de sistematización del proceso a partir de Basagoiti et al. (2001), solo con el ánimo de ejemplificar la lógica de su arquitectura. Con dicha premisa de base, la metodología consistiría en el desarrollo de una serie de fases no necesariamente consecutivas, elaboradas y controladas por los propios actores con el apoyo técnico y teórico de personas especialistas que actuarán como facilitadoras (López, 1993) desempeñando un papel catalítico sin llegar a dominar el proceso (Rahman, 1991).

Las fases que proponen los citados autores, en sintonía con las reportadas en la sistematización publicada por Sirvent $(2018)^{3}$, se sintetizan en cuatro momentos más una evaluación final (Figura 1), además de una etapa previa de arranque que supone la toma de contacto y negociación inicial por parte de las/los promotores o impulsores del proyecto con las autoridades y demás agentes sociales para proponerles su participación en el proceso (Basagoiti et al., 2001).

Figura 1. Fases IAP.

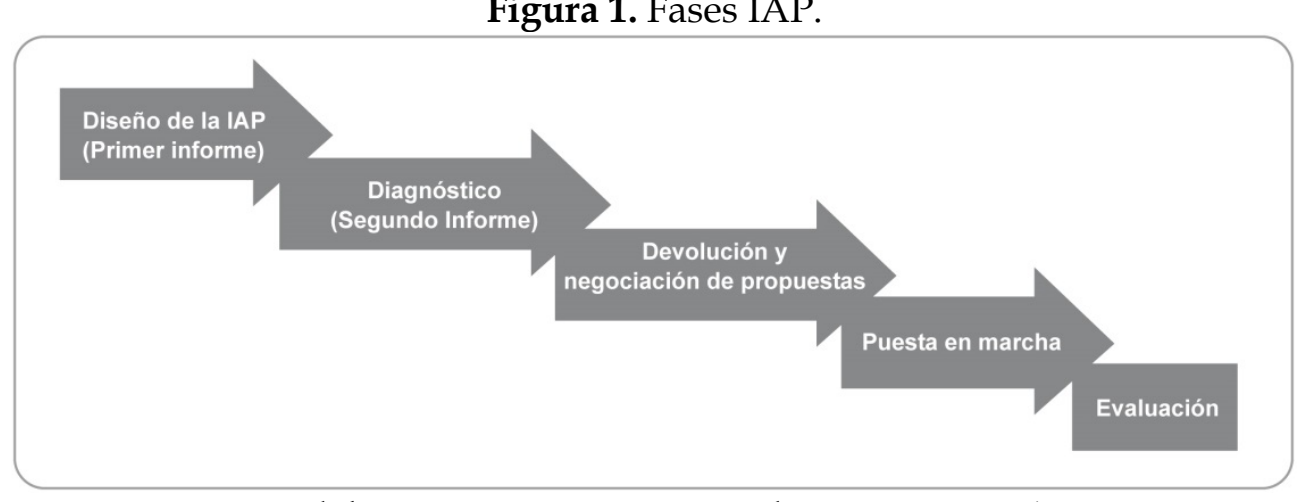

Fuente: Elaboración propia a partir de Basagoiti et al., 2001.

- La primera fase va a consistir en la elaboración de una propuesta de diseño de IAP; para ello se comienza elaborando un anteproyecto, a través de la recogida de información inicial, de las negociaciones con los agentes sociales y la conformación de los grupos de trabajo, a saber, el

${ }^{3}$ A partir de una experiencia de trabajo desarrollada desde 2001 a la fecha en tres barrios de la ciudad de Buenos Aires (Argentina), la autora reporta la validación de un proceso de IAP desarrollado con grupos de líderes e integrantes de organizaciones populares e instituciones educativas, articulado en las siguientes fases: Etapa 1, de conformación del grupo base para la IAP, y Etapa 2, de implementación de la IAP; a su vez, de esta segunda etapa se desprenden cuatro momentos: a) elaboración colectiva del diseño de investigación, b) implementación colectiva del trabajo en terreno de obtención y análisis de la información empírica, c) Planificación e implementación colectiva de sesiones de retroalimentación ampliadas a diversos actores interesados, desarrollándose un "gran Encuentro Comunitario de Retroalimentación", y d) Evaluación del Gran Encuentro Comunitario de Retroalimentación y acciones para la elaboración colectiva del plan de acción (Sirvent, 2018, pp. 21-25).

Prospectiva. Revista de Trabajo Social e intervención social • No. $29 \bullet$ ene.-jun. $2020 \bullet$ pp. 173-192 e-ISSN: 2389-993X • Doi: 10.25100/prts.v0i29.8224

Universidad del Valle - Cali, Colombia 
grupo de IAP (GIAP), un equipo mixto de investigación y trabajo, estable y permanente, formado por vecinos/as, voluntariado y técnicos/as; y la Comisión de seguimiento del proyecto (CS), que es una reunión en la que participan las/los promotores de la investigación, miembros de GIAP y si es posible los organismos públicos implicados, donde se informa sobre el desarrollo del proceso de investigación (Basagoiti et al., 2001). El resultado de esta fase será un primer informe o proyecto de diseño de la IAP que será sometido a discusión.

- $\quad$ En una segunda fase, si el proyecto es aprobado, se comienza con el diagnóstico participado por los actores; en esta fase se desarrolla el trabajo de campo donde se van a emplear diversas técnicas de recogida de información, tanto cualitativas como cuantitativas, como por ejemplo: observación participante, entrevistas individuales y grupales, encuestas, sociograma (mapa social o mapa de relaciones), análisis DAFO. FalsBorda (1991; 1993) propone las siguientes técnicas: la investigación colectiva o de grupos; la recuperación histórica; la valoración y utilización de elementos de la cultura popular; y la comunicación multivocal de los resultados de los trabajos. En esta fase se continúa con la recogida de información y se analizan los datos, igualmente de manera participada, elaborando con los resultados obtenidos un diagnóstico participado de la situación. Esta fase culmina con un segundo informe.

- La tercera fase consistirá en la devolución a la comunidad de los resultados del diagnóstico y en base al mismo, la elaboración de las propuestas de acción. Aquí los actores trabajarán en la elaboración y negociación de dichas propuestas. Para ello se pueden utilizar igualmente diversas técnicas de trabajo y de toma de decisión colectivos, por ejemplo: asambleas participativas (fórum comunitario), mesas de trabajo y dinámicas como el brainstorming o similares. Las propuestas de acción se dirigirán a tres segmentos: (1) a los grupos de poder, (2) a las organizaciones y los movimientos sociales que integran la comunidad, y (3) a la población o ciudadanía (Basagoiti et al., 2001). De esta fase se obtendrá la planificación y programación de las actividades o acciones, al tiempo que se elaborará el diseño de la evaluación de seguimiento y resultados, estableciendo los indicadores adecuados para tal fin.

- La cuarta etapa, y última, consistirá en la puesta en marcha o ejecución del proyecto por las respectivas partes, de la cual se realizará un seguimiento y evaluación final, cuyo resultado será una memoria o informe final. En la Figura 2 se resume todo este proceso. 
Figura 2. Resumen Fases IAP ampliada.

Diseño de Proyecto de IAP

(Entidad promotora)

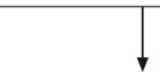

Anteproyecto

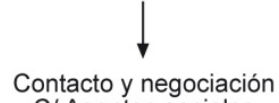

$\mathrm{C} /$ Agentes sociales

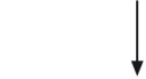

Difusión del Proyecto

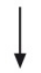

Primer Informe (Proyecto definitivo)
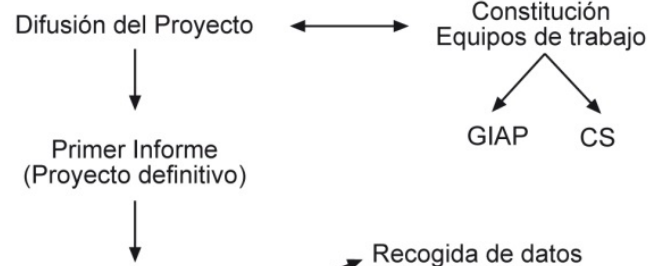

Trabajo de campo

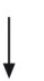

Diagnóstico y propuestas de acción
(Segundo Informe)

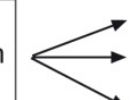

Hacia arriba (instancias de poder)

Hacia el lado (Organizaciones y movimientos sociales)

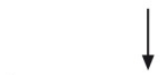

Reunión devolución resultados

Hacia abajo (población/ciudadanía)

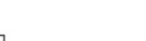

Programacion acciones

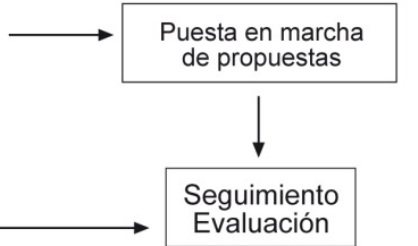

Diseño de evaluación

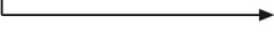

Evaluación

Memoria

Fuente: Elaboración propia a partir de Basagoiti et al., 2001.

\section{El punto de convergencia}

Si toda la trayectoria de la vida de las personas se halla inserta en estructuras sociales que son susceptibles de cambios, en la medida en que dichas estructuras son configuradas por las propias personas, la acción colectiva es, entonces, capaz de producir transformaciones sociales; es en esa idea en la que se basa el fundamento del Trabajo Social Comunitario, el cual para Zamanillo y Martin (2011) "es lo más genuinamente político de todas las dimensiones que ha abordado el trabajo social a lo largo de su historia" (p. 111). Además, prosiguen las autoras, los conceptos fundamentales del Trabajo Social -derivados de los derechos humanos- de autonomía, libertad y dignidad, no tendrían sentido en la 
actualidad si no están atravesados por la noción de emancipación (Zamanillo y Martin, 2011). Por ello, como subraya Duarte (2012) la acción comunitaria es política y pedagógica, pues se orienta al fortalecimiento de la autonomía de los sujetos que integran las comunidades mediante la promoción de la acción colectiva.

Si aceptamos la premisa de que "el conocimiento sirve al sujeto para lograr su emancipación [de cara a] a lograr cambios en las estructuras de poder" (Zamanillo, 1993, p. 229-230), en el plano de los derechos humanos la misma es indiscutible. En los epígrafes precedentes hemos visto que una de las principales barreras para la autonomía de los sujetos, y por ende para la realización de los derechos humanos, es el desconocimiento/desinformación de las personas de sus derechos, de los medios para su defensa y las vías para su exigencia, por lo cual la EDH se constituye en una herramienta indispensable para garantizar dicho conocimiento y la toma de conciencia orientada a la acción.

No obstante, para que los derechos humanos puedan ser legítimamente universales como una aspiración moral y política, y para que sus principios puedan ser comprendidos, luego conocidos, por cualquier persona, deben ser traducidos de acuerdo a los propios códigos de cada cultura con el objeto de propiciar una concepción negociada de los mismos. Ello sobre todo si consideramos que el Trabajo Social se está abriendo en las últimas décadas a un doble proceso, de internacionalización, principalmente a través del ámbito de la cooperación internacional como a través de la nativización, mediante el trabajo con comunidades indígenas. Como señalan Lorente y Zambrano (2010, p. 87) "el Trabajo Social es sensible a la fuerza cultural, moral y política de los pueblos indígenas", lo cual además está reflejado en la propia definición global de la profesión donde se reconocen, dentro de nuestro corpus teórico, los conocimientos indígenas (Federación Internacional de Trabajadores Sociales-IFSW, 2014).

En ese sentido, son muy valiosas las propuestas de diversos autores que abogan por la traducción cultural en el ámbito de la educación y las prácticas en materia de derechos humanos (Allegritti \& Gray, 2005; Calma \& Priday, 2011; Engle, 2013). Para que los conceptos de los derechos humanos sean adoptados localmente, es decir, para poder aterrizar los derechos humanos al plano de lo cotidiano y poder explorar sus posibilidades de realización efectiva en las comunidades, estos deben ser presentados dentro de marcos simbólicos familiares por lo cual la traducción cultural de los mismos se torna indispensable. Esta traducción se ha de realizar siempre sobre la base de los propios códigos culturales y, en el caso de poblaciones indígenas, de su cosmovisión, esto es, de su manera de ver, de interpretar y de actuar sobre el mundo según su experiencia existencial e histórica (Fernández, 2016).

Al-Daraweesh \& Snauwaert (2013) proponen desarrollar la EDH a través de un método hermenéutico. Este método revaloriza la polisemia de las palabras, en toda su complejidad evitando atribuirles un significado único (Cordero, 2011); así, a través de esta metodología interpretativa, los citados autores proponen buscar "equivalentes isomórficos" de los derechos humanos, es decir, equivalentes de los aspectos morales o principios de los derechos humanos que se encuentren codificados en el sistema moral y cultural específico de cada cultura o contexto. De este modo comparan, por ejemplo, los principios de los 
derechos humanos con los principios del budismo y del confucionismo, identificando valores equivalentes que luego transforman en enunciados compatibles con los derechos humanos (Al-Daraweesh \& Snauwaert, 2013).

Entre las prácticas de traducción cultural desarrolladas en el ámbito del Trabajo Social se pueden citar un par de ejemplos. Engle (2013) relata una experiencia de Trabajo Social desarrollada en Hong Kong con el objetivo de desarrollar un programa local de tratamiento de agresores basado en los valores de la masculinidad china "importando" un programa desarrollado en EE.UU., para lo cual se exploraron las concepciones chinas de honor, familia, relaciones y logros, ideas que dificultan que los hombres hablen entre ellos sobre sus problemas, tomen conciencia de que tienen un problema y busquen ayuda para superar su violencia. Así mismo, en Myamar (Birmania) la Fundación Dulwich y la Agencia Australiana para el desarrollo de las mujeres (International Women's Development Agency, IWDA) colaboraron en la elaboración de formas narrativas culturalmente resonantes para trabajar con grupos y comunidades de mujeres en la frontera entre Tailandia y Birmania, víctimas de abusos de derechos humanos, trabajando en conjunto en la construcción de un significado colectivo de sus historias de abuso y miedo (Costello \& Aung, 2015).

Sin embargo, pese a lo atractivo que pueda parecer el proceso de traducción cultural, éste puede llegar a constituirse en un acto de poder, especialmente cuando implica reinterpretar un conjunto de experiencias y categorías en los términos de quien está situado en una posición de poder (Engle, 2013); por ello la IAP se presenta como un método conveniente para llevar a cabo dichos procesos al permitir que se efectúen mediante métodos participativos y negociados, procesos que deben proseguir, además, con el diagnóstico de las vulneraciones de derechos que afectan a la población y sus consecuentes propuestas de acción transformativa. En todo caso, la IAP tampoco estaría exenta de riesgos puesto que el proceso de intervención social mediante prácticas en principio transformadoras, se puede desviar hacia prácticas autoritarias y unilaterales cuando se antepone el conocimiento institucional aplicándose de manera directiva (Lara, 2019).

Siguiendo a López (1993) se puede subrayar la utilidad de la IAP como herramienta para alcanzar el objetivo emancipador de la práctica de Trabajo Social Comunitario y de la $\mathrm{EDH}$, especialmente siguiendo el modelo de la transformación, en tanto permite: (1) acrecentar los conocimientos, al añadir la visión de las poblaciones en sus propios términos, y contrastarlos; (2) la toma de conciencia por parte de las poblaciones de su situación, tanto de las limitaciones o condicionamientos objetivos que les afectan, como de las percepciones subjetivas de los mismos, es decir, cómo perciben dicha situación en los planos micro, meso y macro, qué están dispuestos a -o pueden- hacer para transformarla y qué resultados esperan; (3) el aprendizaje es significativo, en tanto serán los propios sujetos quienes van a generar conocimiento o sistematizar los conocimientos colectivos; (4) el refuerzo e incremento de las relaciones y de las redes sociales, especialmente las redes de información, de intercambio y de presión o lobbies; (5) por último, la movilización colectiva que parte de la acción y la promueve.

Hasta aquí, hemos visto cómo la EDH, IAP y Trabajo Social Comunitario comparten el objetivo emancipador de las personas mediante su toma de conciencia y su participación activa en procesos de transformación de su realidad social. Esta convergencia no es casual.

Prospectiva. Revista de Trabajo Social e intervención social • No. $29 \bullet$ ene.-jun. $2020 \bullet$ pp. 173-192 e-ISSN: 2389-993X • Doi: 10.25100/prts.v0i29.8224

Universidad del Valle - Cali, Colombia 
Los primeros estudios IAP innovadores se desarrollaron entre los años 60 y 70, en el mismo momento en que las teorías críticas y los movimientos de cambio social ya estaban impregnando las ciencias sociales y, en particular, el trabajo social latinoamericano (Duarte, 2017; Healy, 2001). Igualmente, como se ha descrito arriba, en el mismo escenario podemos situar las primeras acciones de EDH; por tanto es evidente que tanto EDH, IAP y Trabajo Social Comunitario no solo comparten un objetivo común, sino también una raíz epistemológica, teórica y metodológica.

Para concluir, y esperando no disuadir de la utilización de prácticas participativas sino solo propiciar la reflexión constructiva, cabe reseñar los desafíos que la inclusión de la IAP y la EDH en el contexto de trabajo con comunidades, tienen por delante.

Siguiendo a Healy (2001) se puede destacar que el estado marginal de las/los trabajadores sociales como investigadoras/es representa un obstáculo para el uso de IAP, por cuanto por un lado para quienes están en la academia implica en cierto modo renunciar al estatus de investigador científico, y por otro, quienes están en la práctica profesional están lejos aún de considerarse siquiera investigadoras/es. Así mismo, añade que en el marco de las administraciones o instituciones, condicionadas por las reglas del mercado, las tareas de las/los trabajadoras/es sociales se reducen muchas veces a la intervención con casos individuales o familias actuando en coordinación con organizaciones externas; en ese contexto, la investigación no se considera una tarea central del Trabajo Social sino más bien instrumental. Por último, la circularidad de los procesos participativos de investigación, el tiempo intensivo y los recursos que requieren, así como los resultados que solo son perceptibles a medio-largo plazo, lo ponen en una desventaja competitiva en relación con la eficiencia económica y la medición de resultados cortoplacistas requeridos en las instituciones burocráticas en cuyo seno se insertan habitualmente estos profesionales.

Por su parte, Duarte (2017) se expresa en los mismos términos cuando, entre otros aspectos, advierte de las limitaciones que significan las políticas públicas al trabajo con comunidades en Chile, en el marco de un modelo neoliberal y dada la consecuente exclusión social que condicionan las intervenciones, así como del riesgo de la instrumentalización de la participación ciudadana. Ello es reafirmado por Morales y Rebollo (2014) para quienes, según su experiencia, los procesos comunitarios como estrategias empoderadoras suelen adoptar sesgos tecnocráticos, prestándose más atención al rigor metodológico en el seguimiento de los pasos y a los resultados de los indicadores, que al sentido político de la propia práctica empoderadora y transformadora, clave en dichos procesos. Así mismo, Lara (2019) a través de la exposición de dos casos desarrollados en México, concluye que las prácticas transformadoras se pueden desviar a prácticas autoritarias y verticales por parte de las instituciones y autoridades, que anteponen y aplican el conocimiento institucional de manera directiva, pues los actores que participan de estos procesos parten evidentemente de una posición de poder inferior; por ello, incide en que es preciso comprender cómo se sostiene y se normaliza en la sociedad la lógica clientelar dentro de los espacios, así como reconocer la desconfianza que tiene la población en la ley y en las instituciones. 


\section{Conclusiones}

En este trabajo se han explorado argumentos teóricos y evidencias que nos permiten concluir que la educación en materia de derechos humanos y la Investigación Acción Participativa pueden converger en acciones comunitarias orientadas a la transformación de las realidades sociales que afectan a ciertas comunidades o colectivos marginados, en tanto comparten no solo un objetivo emancipador, sino también: (1) una base epistemológica, según la cual no es posible conocer la realidad de manera absoluta, objetiva e independiente de sus actores, sino que solo podremos aproximarnos a ella a través de la visión de los propios actores o sujetos en la medida en que estos participen en la construcción de su significado; (2) una base teórica común, que sobre la base de la epistemología referida, se ha venido desarrollando, principalmente, por la Teoría Crítica de la Escuela de Frankfurt en Europa y la educación popular en Latinoamérica; y (3) una base metodológica, cuyo pilar constituye la participación activa de las personas en la toma de conciencia de su situación y en el desarrollo y ejecución de acciones encaminadas a transformar aquellas realidades injustas, estrechamente vinculadas con la vulneración de sus derechos.

Por su parte, el Trabajo Social, aunque puede diferir en lo epistemológico al desarrollarse desde epistemologías muy diversas dependiendo de cada contexto geográfico y ámbito de actuación, y pudiendo diferir asimismo en lo teórico (consecuencia de dicha diversidad), como práctica comunitaria comparte con la EDH y la IAP, el objetivo emancipador. Asimismo, comparte la convicción de que este solo puede alcanzarse con la implicación activa de los sujetos, esto es su participación, su concienciación y su acción. Sin embargo, parafraseando las conclusiones derivadas de las experiencias documentadas por Lara (2019), quienes trabajamos por la justicia social debemos mantener constante la reflexividad en nuestras actuaciones, ya sea que se desarrollen desde las academias, las instituciones públicas o las organizaciones no gubernamentales, intentando evitar rigideces y universalismos autoritarios.

\section{Referencias bibliográficas}

Adorno, T. (1998). Educación para la emancipación. Conferencias y conversaciones con Hellmut Becker (1959-1969). Madrid: Morata.

Alberich, T. (2008). IAP, Redes Y Mapas Sociales: Desde la Investigación a la Intervención Social. Portularia: Revista de Trabajo Social, 8(1), 131-151.

Al-Daraweesh, F., \& Snauwaert, D. (2013). Toward a Hermeneutical Theory of International Human Rights Education. Educational Theory, 63(4), 389-411.

Allegritti, I., \& Gray, M. (2005). Mamphele Ramphele and Xhosa culture: Some insights on culture, self-determination and human rights for South African social work. Social Work/Maatskaplike Werk, 41(2), 131-142.

Ampudia, M. (2012). Movimientos sociales y Educación Popular. Reflexiones sobre la experiencia educativa de los Bachilleratos Populares. Revista osera, (6), 1-17.

Prospectiva. Revista de Trabajo Social e intervención social • No. $29 \bullet$ ene.-jun. 2020 • pp. 173-192 e-ISSN: 2389-993X • Doi: 10.25100/prts.v0i29.8224

Universidad del Valle - Cali, Colombia 
Asamblea General de Naciones Unidas (2017). Mesa redonda sobre la aplicación de la Declaración de las Naciones Unidas sobre Educación y Formación en materia de Derechos Humanos: buenas prácticas y dificultades - Informe resumido de la Oficina del Alto Comisionado de las Naciones Unidas para los Derechos Humanos, A/HRC/35/6. Recuperado de

https:/ / documents-ddsny.un.org/doc/UNDOC/GEN/G17/075/55/PDF/G1707555.pdf?OpenElement.

Bajaj, M. (2011). Human Rights Education: Ideology, Location, and Approaches. Human Rights Quarterly, 33(2), 481-508.

Basagoiti, M., Bru, P., y Lorenzana, C. (2001). IAP de bolsillo. Madrid: Acsur-Las Segovias.

Beydili, E., \& Yildirim, B. (2013). Human Rights as a Dimension of Social Work Education. Procedia - Social and Behavioral Sciences, 106, 1393-1398.

Brito, Z. (2008). Educación popular, cultura e identidad desde la perspectiva de Paulo Freire. En M. Godotti, M. Gomez, J. Mafra, A. Fernandes de Alencar (Comps), Paulo Freire. Contribuciones para la pedagogía (pp. 29-45). Buenos Aires: CLACSO.

Calma, T., \& Priday, E. (2011). Putting indigenous human rights into social work practice. Australian Social Work, 64(2), 147-155.

Claude, R. (2003). Educación Popular en Derechos Humanos: 24 guías de actividades participativas para maestros y facilitadores. San José: Instituto Interamericano de Derechos Humanos, IIDH.

Cordero, N. (2011). Trabajo Social y Hermenéutica Crítica: una opción metodológica para desvelar elementos éticos en los orígenes de la profesión en Sevilla. Portularia. Revista de Trabajo Social, 11(1), 87-97.

Costello, S., \& Aung, U. (2015). Developing social work education in Myanmar. International Social Work, 58(4), 582-594.

Cubillos-Vega, C. (2018). La inclusión de los Derechos Humanos en la agenda del Trabajo Social. Una aproximación a la Educación en Derechos Humanos (EDH) en la formación académica (Tesis de Doctorado). Universidad Complutense de Madrid, Facultad de Trabajo Social. Madrid, España.

Cubillos-Vega, C. (2019). Bienestar social: un objetivo compartido. Sobre la alianza entre los derechos humanos y el trabajo social. Arbor, 195(791), a493. doi: 10.3989/arbor.2019.791n1006.

Cubillos-Vega, C., y Llanán, J. (2018). Pedagogía de la memoria y escritura performativa en la educación en derechos humanos. Revista Latinoamericana de Derechos Humanos, $29(1), 36-50$.

De la Riva, F. (1993). Investigación participativa y autoformación grupal. Documentación Social, 92, 141-152.

Dewey, J. (2002). Democracia y educación. (5 ed.). Madrid: Morata. 
Duarte, C. (2012). Fundamentos ético-políticos del trabajo social comunitario. Debate y Sociedade, 2(3), 69-79.

Duarte, C. (2017). Trabajo social comunitario:perspectivas teóricas, metodológicas, éticas y políticas (Tesis de Doctorado). Universidad Complutense de Madrid, Facultad de Trabajo Social. Madrid, España.

Elisalde, R., y Ampudia, M. (2008). Movimientos sociales y educación: teoría e historia de la educación popular en Argentina y América Latina. Buenos Aires: Buenoslibros.

Engle, S. (2013). Transnational Human Rights and Local Activism: Mapping the Middle. En R. Provost y C. Sheppard, (Eds.), Dialogues on Human Rights and Legal Pluralism (pp. 217-228). London: Springer.

Fals-Borda, O. (1991). Algunos ingredientes básicos. En O. Fals-Borda y M.A. Rahman (Eds.), Acción y conocimiento. Como romper el monopolio con Investigación-Acción Participativa (pp. 7-19). Santafé de Bogotá: CINEP.

Fals-Borda, O. (1993). La investigación participativa y la intervención social. Documentación Social, (92), 9-23.

Federación Internacional de Trabajadores Sociales-IFSW. (2014). Definición global de la profesión de Trabajo Social. Recuperado de https://www.ifsw.org/what-is-socialwork/global-definition-of-social-work/.

Fernández, K. (2016). Paradigma de investigación y pueblos indígenas del primer mundo: crítica cultural del trabajo social. Revista andaluza de antropología, (10), 54-78.

Flowers, N. (2015). The Global Movement for Human Rights Education. Radical Teacher, 103, 5-17. doi: 10.5195/rt.2015.237.

Freire, P. (1990). La naturaleza política de la educación: cultura, poder y liberación. Madrid: Paidós. Ministerio de Educación y Ciencia

Freire, P. (2012). Pedagogía del oprimido. (2 ed.). Madrid: Siglo XXI.

Hawkins, C., \& Knox, K. (2014). Educating for international social work: Human rights leadership. International Social Work, 57(3), 248-257.

Healy, K. (2001). Participatory action research and social work. A critical appraisal. International Social Work, 44(1), 93-105.

Lara, G. (2019). Cuestionar la práctica investigativa: experiencias colaborativas en dos regiones en México. EMPIRIA. Revista de Metodología de Ciencias Sociales, (44), 179-211.

López, P. (1993). La IAP: un enfoque integral. Documentación Social, (92), 71-78.

Lorente, B. y Zambrano, V. (2010). Reflexividad, Trabajo Social comunitario y sensibilización en derechos. Cuadernos de Trabajo Social, (23), 85-102.

Magendzo, A. (2003). Pedagogía Crítica y Educación en Derechos Humanos. Paulo Freire. Revista de Pedagogía Crítica, (2), 19-27. 
Magendzo, A. (2015). Propósitos y desafíos de la educación en derechos humanos en el momento presente. Revista de Derechos Humanos, 10(13), 46-51.

Magendzo, A., y Bermúdez, A. (2018). Pensando la educación en derechos humanos desde una mirada ética y controversial. Revista Latinoamericana de Derechos Humanos, 28(2), 17-34. doi: 10.15359/rldh.28-2.1.

Martínez, S. y Agüero, J. (2008). La dimensión político-ideológica del Trabajo Social. Claves para un trabajo social emancipador. Buenos Aires: Dunken.

Mejía, M. (2011). Educaciones y pedagogías críticas desde el Sur (Cartografías de la Educación Popular). Lima: Viceministerio de Educación Alternativa y Especial.

Mihr, A., \& Schmitz, H. (2007). Human Rights Education (HRE) and Transnational Activism. Human Rights Quarterly, 29(4), 973-93.

Miranda, M. (2003). Pragmatismo, Interaccionismo simbólico y Trabajo Social. De cómo la caridad y la filantropía se hicieron científicas (Tesis de Doctorado). Universitat Rovira I Virgili. Tarragona, España.

Miranda, M. (2014). Prólogo. En J. Addams, El largo camino de la memoria de las mujeres (pp. IX-XX). Zaragoza: Universidad de Zaragoza.

Morales, E., y Rebollo, O. (2014). Potencialidades y límites de la acción comunitaria como estrategia empoderadora en el contexto de crisis actual. Revista de Treball Social RTS, (203), 9-22.

Muñoz, D.A. (2013). Lectura de contexto: La educación popular como práctica libertaria. El Ágora USB. Revista de Ciencias Sociales, 13(1), 349-365. doi: 10.21500/16578031.89.

Nadkarni, V., \& R. Sinha. (2016). Transforming social work education in India: integrating human rights. Journal of Human Rights and Social Work, 1(1), 9-18.

Observatorio Internacional de Ciudadanía y Medio Ambiente Sostenible-CIMAS. (2009). Manual de Metodologías Participativas. Madrid: CIMAS.

Ortiz, M., y Borjas, B. (2008). La Investigación Acción Participativa: aporte de Fals Borda a la educación popular. Espacio abierto, 17(4), 615-627.

Rahman, M.A. (1991). El punto de vista teórico de la IAP. En O. Fals-Borda y M.A. Rahman (Eds.), Acción y conocimiento. Como romper el monopolio con Investigación-Acción Participativa (pp. 21-35). Santafé de Bogotá: CINEP.

Rodríguez, T. (1993). Aportaciones básicas de la IAP a la epistemología y metodología. Documentación Social, (92), 23-43.

Rojas, C. (2015). Fundamentos de la educación en derechos humanos desde la perspectiva crítica: Pedagogía para oprimidos y (des)esperanzados. Revista de Derechos Humanos, 10(13), 53-57.

Sirvent, M.T. (2018). De la educación popular a la investigación acción participativa. Perspectiva pedagógica y validación de sus experiencias. InterCambios. Dilemas y transiciones de la Educación Superior, 5(1), 12-29.

Prospectiva. Revista de Trabajo Social e intervención social • No. $29 \bullet$ ene.jun. 2020 • pp. 173-192

e-ISSN: 2389-993X • Doi: 10.25100/prts.v0i29.8224

Universidad del Valle - Cali, Colombia 
Tibbitts, F. (2002). Understanding what we do: Emerging models for human rights education. International Review of Education, 48(3-4), 159-171.

Tibbitts, F. (2017). Evolution of human rights education models. En M. Bajaj (Ed.), Human Rights Education Theory, Research, Praxis (pp. 69-95). Pennsylvania: University of Pennsylvania Press.

Tuvilla, J. (2008). La educación en derechos humanos en España. Revista de la Asociación de Inspectores de Educación de España, (9), 1-22.

UNESCO. (2000). All human beings... A manual for human rights education. Paris: UNESCO Publishing.

Ventura, C. (2018). Educación popular y liberadora para hacer frente a la crisis de derechos humanos en México. En F. Conde (Ed.), Coloquio Internacional sobre Educación en Derechos Humanos (pp. 23-33). Ciudad de México: Comisión de Derechos Humanos del Distrito Federal.

Zamanillo, T. (1993). La IAP en trabajo social. Documentación Social, (92), 225-240.

Zamanillo, T., y Martín, M. (2011). La Responsabilidad política del Trabajo Social. Trabajo Social Global, 2(3), 97-115.

Zúñiga, R. (2018). Educación popular y la vigencia de los derechos humanos. En F. Conde (Ed.), Coloquio Internacional sobre Educación en Derechos Humanos (pp. 34-41). Ciudad de México: Comisión de Derechos Humanos del Distrito Federal. 


\section{OTROS ARTÍCULOS DE PROSPECTIVA No. 29 DE 2020}

EDITORIAL

La Reconceptualización: una opción a la encrucijada del Trabajo Social en Colombia Víctor Mario Estrada-Ospina

\section{ARTÍCULOS}

Formación profesional y la práctica supervisada en el Servicio Social brasileño

Reginaldo Ghiraldelli

El rol docente del tutor y supervisor de prácticas de Trabajo Social: construcción de la reflexividad y el compromiso durante la formación a través de la supervisión

Carmina Puig-Cruells

Problematizando las prácticas preprofesionales en Trabajo Social. Desafios y perspectivas

Ruth Noemí Parola

Trabajo Social en contextos de formación política: la práctica transformadora

Angélica López-Yepes

Jackeline Cuenca-Echeverry

Investigación cuantitativa en una práctica universitaria de intervención en comunidad en el norte de México

Adán Cano-Aguilar
Interventores y comunidades vulnerables: una propuesta de formación para universitarios

Dulce Nayeli Cohetero-Hernández

Fidel González-Quiñones

Reagrupación familiar de menores en Aragón. Propuestas de acompañamiento en el proceso migratorio

Chabier Gimeno-Monterde

José David Gutiérrez-Sánchez

Aportaciones de la Educación en Derechos Humanos (EDH) y la Investigación Acción Participativa (IAP) en contextos de trabajo comunitario

Carla Cubillos-Vega

Condiciones para una NeoReconceptualización del Trabajo Social en Chile, Latinoamérica y el Caribe

Luis Vivero-Arriagada

Justicia sin daño: una apuesta por el fortalecimiento del acceso a la justicia Diego Fernando Acosta-Daza

\section{IN MEMORIAM}

Camilo Bautista Ochoa, un santandereano que se enamoró del Pacífico

Jesús Glay Mejía-Naranjo

\section{PROSPECTIVA}

Revista de Trabajo Social e Intervención Social 